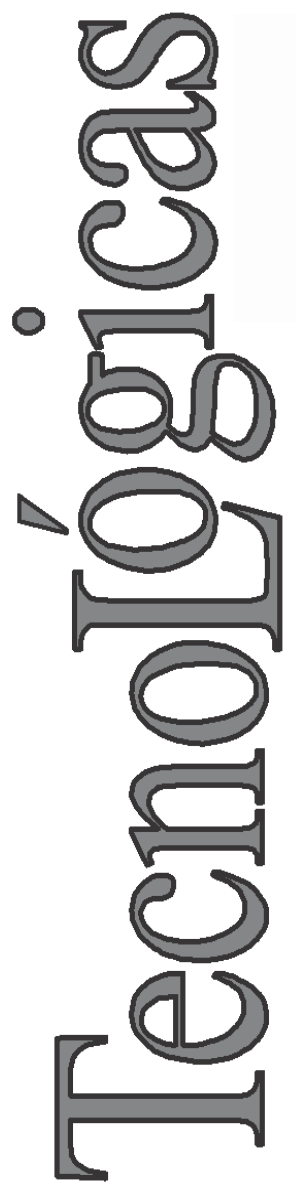

\title{
Simulación de un Sistema de Generación de Campo Eléctrico a Baja Frecuencia para Análisis de Señales Inducidas en Células Cultivadas In Vitro
}

\section{Computation of Low Frequency Electric Field Generation System for Analysis of Induced Signals in In-Vitro Cultured Cells}

Jenniffer Zapata-Giraldo ${ }^{1}$ María E. Moncada-Acevedo ${ }^{2}$ Adolfo Escobar-Ordoñez ${ }^{3}$

1 Estudiante de Ingeniería Biomédica, Instituto Tecnológico Metropolitano, Medellín-Colombia, ferzapatagiraldo@gmail.com

2 Grupo de Investigación en Materiales Avanzados y

Energía, Instituto Tecnológico Metropolitano, Medellín-Colombia mariamoncada@itm.edu.co

3 Grupo de Investigación en Materiales Avanzados y

Energía, Instituto Tecnológico Metropolitano, Medellín-Colombia adolfoescobar@itm.edu.co 


\title{
Resumen
}

Diversos estudios de modelamiento y simulación de reacciones de sistemas celulares y exposición humana ante campos eléctricos externos, han permitido establecer umbrales de exposición, rangos de aplicación terapéutica y niveles experimentales de diferentes variables eléctricas con miras de uso a diferentes patologías y caracterización de mecanismos fisioeléctricos. En este trabajo se desarrolló un modelo computacional 3D conformado por una fuente de campo eléctrico y un contenido celular depositado en una caja tipo Falcom. El modelo consideró las geometrías, propiedades, mallado, condiciones de simulación y tipo de análisis de resultados. El desarrollo fue realizado en la herramienta computacional ANSYS ${ }^{\circledR}$ y con este se logró estimar el comportamiento de la densidad de corriente inducidas en los cultivos celulares a partir de diversas condiciones de estimulación. El modelo creado y los resultados encontrados son un apoyo para la estimación y predicción de la señal eléctrica inducida en los cultivos con la que asociar una respuesta biológica encontrada.

\section{Palabras clave}

Modelo computacional; electrodos paralelos; variables eléctricas inducidas; estimulación eléctrica.

\begin{abstract}
Various modeling and simulation studies about cellular systems reactions and human exposure to external electric fields, have established exposure thresholds, therapeutic application ranges, and different levels of experimental variables in order to use in different pathologies and characterization of physio-electric mechanisms. In this work, we developed a 3D computational model that consists of an electric field source and cell content deposited in a Falcom plate. The model considered the geometries, properties, meshing, simulation conditions, and type of results analysis. The development was performed in the ANSYS ${ }^{\circledR}$ software and it allowed estimate the behavior of the induced current density in cell cultures from various stimulation conditions. The developed model and the results are supportive of the estimation and prediction of the electrical signal induced in cells with which to associate a biological response.
\end{abstract}

\section{Keywords}

Computational model; parallel electrodes; electrical variables induced; electric stimulation. 


\section{INTRODUCCIÓN}

Emil Du Bois-Reymond uno de los fundadores de la electrofisiología, fue quien documento en detalle actividades eléctricas asociadas con excitación nerviosa, contracción muscular y procesos de cicatrización (Du Bois-Reymond, 1843). Actualmente es conocido que los campos eléctricos (CE) endógenos, están presentes en los organismos vivos, direccionando e influyendo en diversos procesos biológicos (Kloth, 2005; Zhao et al., 2004).

A nivel celular, ante la aplicación de campos eléctricos externos se han encontrado incrementos en la circulación y migración de células epiteliales (Zhao et al., 2002), neutrófilos, macrófagos y diversos factores de crecimiento (Zhao, 2006); además de aumentar la producción de fibroblastos, los niveles de colagenasa y de disminuir el edema e inhibir el surgimiento de mastocitos y bacterias (Bagnaninchi \& Drummond, 2011). Se ha logrado también la recuperación de células epiteliales de la córnea y mejoras en la velocidad y direccionalidad de la migración de fibroblastos 3T3 en heridas (Song et al., 2002). Otros estudios presentan la aplicación de campos eléctricos externos como la base para la transfección de material genético (Neumann et al., 1999) y para la administración de fármacos, incluyendo su uso en el tratamiento de cáncer a través de electro-quimioterapia (Belehradek et al., 1993; Tsong, 1991). Ante anormalidades esqueléticas y neuronales se ha encontrado la presencia de gradientes eléctricos. Sin embargo, el efecto en el tejido depende del tipo de campo aplicado cuya señal puede cambiar en magnitud, frecuencia, tiempo de exposición, tipo de onda entre otros.

En relación a los modelos y simulaciones computacionales, con estos se han logrado estimar umbrales de densidad de corriente para activación de células a lo largo del rango de frecuencia de $\mathrm{kHz}$ (Bohnert \& Dössel, 2010). Igualmente, se ha llegado a implementar un Modelo de Ambiente de Desarrollo (MDE) celular en 2D para estudiar el proceso de transporte iónico, la anisotropía local alrededor de las membranas celulares, las interfaces biológicas y el comportamiento dispersivo de los tejidos permitiendo la introducción de una nueva forma geométrica de aproximación con polígonos que muestra mejores resultados que las formas tradiciona- 
les (círculos, elipses o cuadrados), demostrando la importancia de las distribuciones de potenciales de las células biológicas (Suzuki et al., 2007).

En este trabajo se desarrolla un modelo computación para la simulación de un sistema de campo eléctrico a baja frecuencia con el cual estimar las variables eléctricas que son inducidas en células cultivadas in vitro, también representadas en el modelo. El trabajo es realizado en el programa Ansys ${ }^{\circledR}$, en su módulo Workbench y APDL. Las partes del sistema son construidas en 3D y es evaluada la señal generada por la fuente de estimulación y luego la misma señal ante la presencia del material celular. Los resultados presentan un comportamiento estable de la señal generada por la fuente y la distribución que de las señales se hace al interior de la representación celular.

\section{METODOLOGía}

A continuación se presenta la metodología de diseño y construcción del modelo computacional para la estimulación de células con campo eléctrico. Igualmente, se describe la metodología de inclusión del contenido celular y las condiciones de simulación que llevaron a estimar las señales inducidas ante la estimulación. Para el análisis eléctrico fue necesario establecer para el modelo creado: geometría, propiedades, mallado, condiciones de simulación y análisis de resultados. El modelo fue creado con la herramienta computacional ANSYS $₫$, con cuya licencia cuenta el Instituto Tecnológico Metropolitano. Los tres modelos conteniendo la fuente, la caja de cultivo y los volúmenes representativos de las células se construyeron en APDL Ansys ${ }^{\circledR}$.

La geometría del sistema electrodos-células fue construida en 3D y está conformada por cuatro partes: a) Las placas paralelas (electrodos), b) la caja Falcom de 24 pozos, c) los cilindros macizos que representan el contenido celular y d) una esfera de potencial cero conteniendo las geometrías anteriores. Cada parte corresponde con las dimensiones reales del sistema experimental y la esfera es 3 veces el tamaño de los electrodos de acuerdo a lo recomendado para garantizar un potencial de referencia lo suficientemente 
lejano. La Fig. 1 muestra la geometría creada para el sistema electrodos-células.

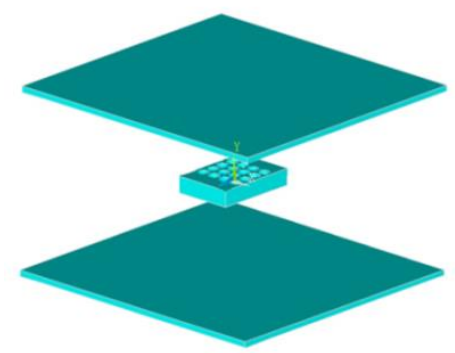

Fig. 1. Geometría para el sistema electrodos-células. Fuente: Autores

Para el análisis eléctrico fueron utilizadas las propiedades de resistividad eléctrica y permitividad eléctrica. Los valores de resistividad para las células fueron tomados de un trabajo previo y la permitividad eléctrica fue tomada de literatura equivalente a tejido biológico humano. Los valores para los otros volúmenes fueron establecidos de acuerdo al tipo de material. Para la caja Falcom fue utilizado el poliestireno, para los electrodos el aluminio y para la esfera el del aire. Las propiedades fueron consideradas isotrópicas para cada uno de los volúmenes.

Una vez establecida la geometría del sistema, con sus elementos y materiales fue creada la malla sobre la cual se establecen los elementos que llevarán a la solución de las ecuaciones de Maxwell. En el modelo fueron utilizados dos formas de mallado: para los electrodos dado su simetría, fueron utilizados elementos tetraédricos que permiten fácil y rápida solución y para los otros volúmenes fueron utilizados elementos hexaédricos dada sus formas irregulares.

Posterior a la creación de la malla, las placas paralelas que representan los electrodos fueron cargadas con el potencial eléctrico a una frecuencia definida. El elemento escogido para este fue el SOLID122 que permite análisis de carga, con un grado de libertad de voltaje y es aplicable para modelos electrostáticos en 3D y para análisis tiempo-armónicos de campo eléctrico cuasi estáticos. Uno de los electrodos fue cargado con voltaje entre $30 \mathrm{~V}$ y $150 \mathrm{~V}$ y el otro a potencial cero. La esfera fue establecida para condición de 
frontera y cargada con voltaje cero. La Fig. 2 muestra el sistema con las cargas asignadas.

El método de solución escogido fue el FULL (método completo). Para solucionar las ecuaciones (EQSLV) se utilizó el método directo, aplicable a valores reales o valores complejos para matrices simétricas y asimétricas. Este método solo está disponible para los tipos de análisis (ANTYPE) Estático, Armónico (solo para el método completo utilizado en el análisis) y Transitorio. En el modelo armónico fue necesario también definir la frecuencia a través de las opciones HARFRQ: la cual define el rango de frecuencia en la respuesta del análisis armónico. Previa a la evaluación del modelo completo, fue realizada una evaluación de los electrodos con la caja Falcom sin contenido celular para observar las diferentes acciones del campo eléctrico ante esta condición.

\section{RESULTADOS Y DISCUSIÓN}

En primer lugar se evaluó el voltaje (o potencial eléctrico) y el campo eléctrico inducido en los electrodos. La Fig. 2 muestra la distribución del voltaje y del campo eléctrico en las placas paralelas. La distribución de estas variables en los electrodos se conserva para las diferentes condiciones simuladas. Se puede observar, en cuanto al campo eléctrico, que los máximos valores se presentan en las esquinas y los lados de los electrodos debido a la concentración de cargas eléctricas en estos puntos por el efecto borde.

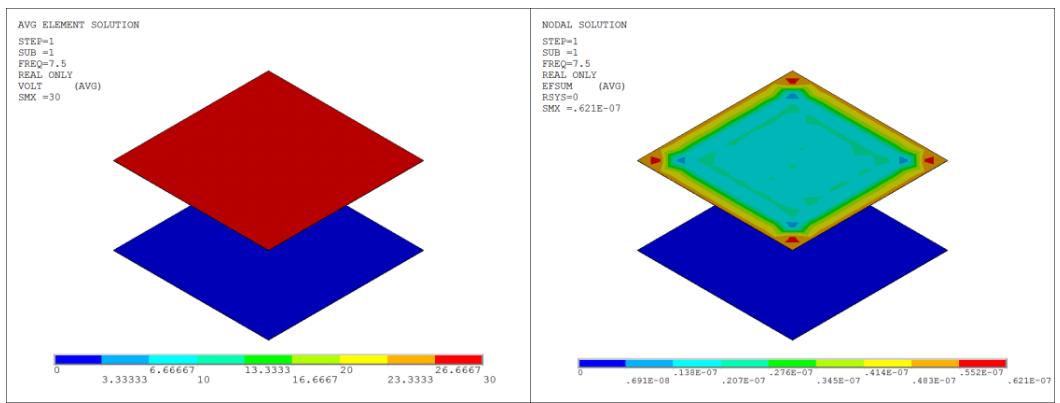

(a) (b)

Fig. 2. Distribución de a) voltaje y b) campo eléctrico en los electrodos. Fuente: Autores 
Posteriormente, y antes de analizar la inducción de variables en el sistema electrodos-células completo, se evaluó la distorsión del campo eléctrico en el centro del arreglo, al contar con la caja Falcom sin el material celular (vacía) y con el material celular en los cuatro pozos del centro. La Fig. 3 muestra la distribución de voltaje en un plano que pasa por el centro del arreglo y es perpendicular a las placas. La Fig. 4 muestra la distribución de campo eléctrico en un perfil que pasa por el centro del arreglo y es paralelo a las placas (a nivel de la caja y las células).

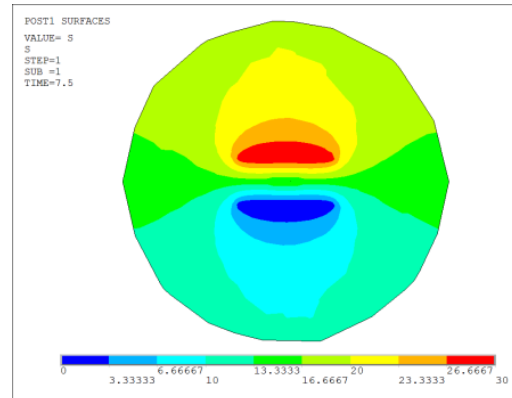

a) Sin material celular

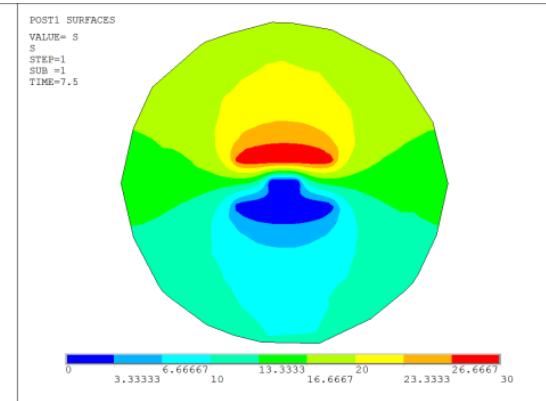

b) Con material celular

Fig. 3. Comportamiento del voltaje en el centro del sistema. Fuente: Autores

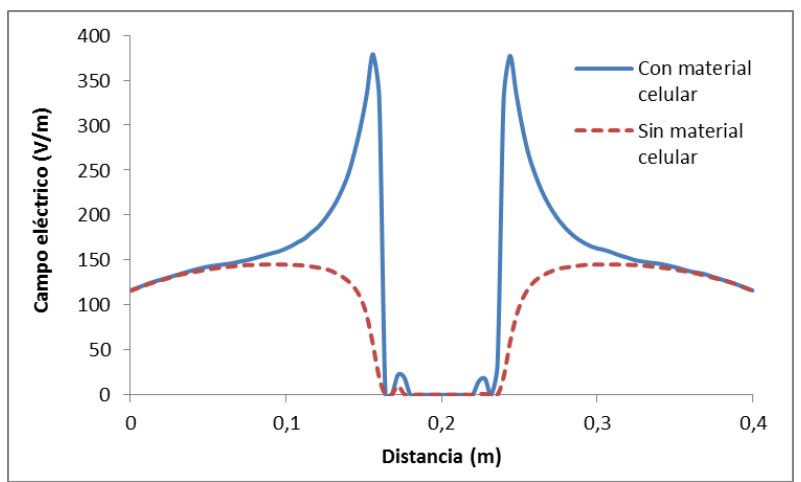

Fig. 4. Comportamiento del campo eléctrico en el centro del sistema.

Fuente: Autores

El voltaje no cambia ante la introducción de la caja Falcom al sistema conservando un valor de $15 \mathrm{~V}$ a nivel del plano en el cen- 
tro entre los electrodos, lo que es de esperarse pues no posee materiales conductores en su interior. Por otro lado, se observa como el campo eléctrico en el centro del arreglo cae abruptamente al ingresar la caja Falcom de acuerdo al efecto de no uniformidad que hace que se presente una señal de salida más baja que la esperada. Al introducir el material celular, el voltaje inducido en el centro de los electrodos a lo largo de la caja Falcom, cae abruptamente a causa del efecto de no continuidad. El campo eléctrico por su parte, se comporta de acuerdo a los dos efectos conocidos como proximidad y no uniformidad. El efecto de proximidad hace que ante la presencia de un material conductor como lo son las células, se perturbe la distribución de carga original en los electrodos que generan el campo, introduciendo una imagen dipolo que refuerza el campo original cerca del objeto conductor y generando un error positivo. El de no uniformidad por el contrario ocasiona una caída abrupta del campo eléctrico a lo largo del material conductor.

Por último, se evaluó el potencial eléctrico, el campo eléctrico y la densidad de corriente inducida en el material celular para diferentes niveles de voltaje aplicado en los electrodos y a diferentes valores de frecuencia. La Fig. 5 muestra la densidad de corriente inducida en la caja y las células para un nivel de tensión de $30 \mathrm{~V}$ y a $7,5 \mathrm{~Hz}$.

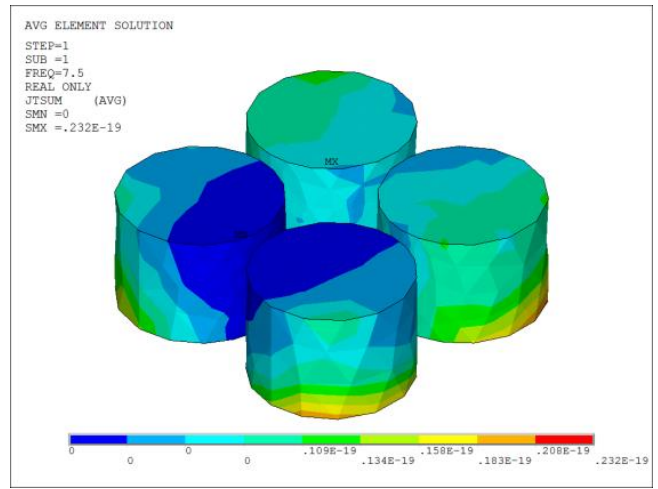

Fig. 5. Densidad de corriente inducida en el material celular. Fuente: Autores 


\section{CONCLUSIONES}

El trabajo realizado permitió la construcción de un modelo computacional con un par de electrodos paralelos entre sí como fuente de estimulación de campo eléctrico. El tamaño de los electrodos garantizó un área de campo homogéneo en el centro de la caja de cultivo celular y los resultados de campo eléctrico encontrado están acorde con el comportamiento esperado.

El modelo computacional entregado incluye la representación de un cultivo celular depositado sobre una caja Falcom. Con este modelo y a partir de propiedades de resistividad obtenidas de un estudio previo, se logró estimar la magnitud de diversas variables eléctricas generadas por la estimulación con campos eléctricos y que son importantes a la hora de decidir experimentación celular. El modelo 3D para estimulación con campo eléctrico permitió observar los fenómenos de proximidad y no uniformidad, presentes en un campo eléctrico perturbado por un objeto y un objeto conductor. Estos fenómenos pueden asociarse a las diferentes pendientes presentadas en los resultados de estimulación por campo eléctrico ante el cambio de frecuencia.

Dentro de los estudios de experimentación in vitro e in vivo, relacionados con estimulación por campos eléctricos, la literatura continúa reportando cada vez más aplicaciones a diferentes patologías. Sin embargo, el amplio rango de posibilidades en frecuencia, magnitudes, tiempos de estimulación, diferencias en las poblaciones, entre otras, sigue dificultando crear estándares que diferencien su aplicación en cada patología específica. El desarrollo de modelos computacionales con los que estimar las variables eléctricas que se están induciendo ahorrará tiempo de investigación y dinero.

\section{REFERENCIAS}

ABagnaninchi, P. O., \& Drummond, N. (2011). Real-time label-free monitoring of adipose-derived stem cell differentiation with electric cellsubstrate impedance sensing. Proceedings of the National Academy of Sciences of the United States of America, 108(16), 6462-7. 
Belehradek, M., Domenge, C., Luboinski, B., Orlowski, S., Belehradek, J., \& Mir, L. M. (1993). Electrochemotherapy, a new antitumor treatment. First clinical phase I-II trial. Cancer, 72(12), 3694-700.

Bohnert, J., \& Dössel, O. (2010). Effects of time varying currents and magnetic fields in the frequency range of $1 \mathrm{kHz}$ to $1 \mathrm{MHz}$ to the human body - a simulation study. Annual International Conference of the IEEE Engineering in Medicine and Biology Society, 2010, 6805-8.

Du Bois-Reymond, E. (1843). Vorläufiger Abriss einer Untersuchung über den sogenannten Froschstrom und über die elektromotorischen Fische. Annalen der Physik und Chemie, 134(1), 1-30.

Kloth, L. C. (2005). Electrical stimulation for wound healing: a review of evidence from in vitro studies, animal experiments, and clinical trials. The international journal of lower extremity wounds, 4(1), 23-44.

Neumann, E., Kakorin, S., \& Toensing, K. (1999). Fundamentals of electroporative delivery of drugs and genes. Bioelectrochemistry and bioenergetics, 48(1), 3-16.

Song, B., Zhao, M., Forrester, J. V, \& McCaig, C. D. (2002). Electrical cues regulate the orientation and frequency of cell division and the rate of wound healing in vivo. Proceedings of the National Academy of Sciences of the United States of America, 99(21), 13577-82.

Suzuki, D. O. H., Ramos, A., \& Marques, J. L. B. (2007). Modeling environment for numerical simulation of applied electric fields on biological cells. Electromagnetic biology and medicine, 26(3), 239-50.

Tsong, T. Y. (1991). Electroporation of cell membranes. Biophysical journal, 60(2), 297-306.

Zhao, M. (2006). Electrical Stimulation and Angiogenesis. In D. Janigro (Ed.), The Cell Cycle in the Central Nervous System (pp. 495-509). Humana Press.

Zhao, M., Bai, H., Wang, E., Forrester, J. V, \& McCaig, C. D. (2004). Electrical stimulation directly induces pre-angiogenic responses in vascular endothelial cells by signaling through VEGF receptors. Journal of cell science, 117(Pt 3), 397-405.

Zhao, M., Pu, J., Forrester, J. V, \& McCaig, C. D. (2002). Membrane lipids, EGF receptors, and intracellular signals colocalize and are polarized in epithelial cells moving directionally in a physiological electric field. FASEB journal, 16(8), 857-9. 